\title{
Development of the knowledge test of medical instruments and materials for cancer chemotherapy
}

Kouichi Tanabe ( $\nabla$ tanabeko-mi@umin.ac.jp )

University of Toyama

Atsumi Nitta

University of Toyama

Hideki Origasa

University of Toyama, University of Toyama

Miyuki Nishitani

University of Toyama

Miki Yatsuduka

University of Toyama

Tomomi Yasuda

University of Toyama

Hatsuna Yasuda

University of Southampton

Kenichi Hosoya

University of Toyama

\section{Research Article}

Keywords: medical instruments, medical materials, cancer chemotherapy, pharmaceutical education, pharmacopedia, palliative medicine

Posted Date: December 14th, 2021

DOl: https://doi.org/10.21203/rs.3.rs-1007696/v2

License: (c) This work is licensed under a Creative Commons Attribution 4.0 International License. Read Full License 


\section{Abstract}

Background: Focusing on medical instruments and materials used for high-risk medicines for cancer treatments, a test on knowledge of medical instruments requiring acquisition of basic knowledge and materials was developed for students in pharmacy school. The aim of the study is to investigate the reliability and validity of the test (medical instruments and materials for cancer treatment-Questionnaire 45; MIMCT-Q45) we developed.

Patients and Methods: Focus group discussion was performed by participants consisting of medical staff considered to have abundant experience of cancer chemotherapy. Content analysis was performed and a list of extracted medical instruments and materials was prepared. A questionnaire survey was performed twice in pharmacy students to confirm reliability employing the retest method. Responses were also collected from nurses and pharmacists to investigate discriminative validity on comparison with the students. Furthermore, difficulty and discrimination were estimated using the item response theory (IRT).

Results: Thirteen types of medical instruments and materials were extracted and listed in the knowledge test. In the questionnaire survey, the overall Cronbach's a and interclass correlation coefficient were high, but Cronbach's a was slightly low (0.56-0.58) in some categories. The range of discrimination estimated based on IRT was 0.98-3.09, and that of difficulty was $-0.91-3.00$.

Conclusions: A knowledge test on cancer chemotherapy-related medical instruments and materials including palliative care at home (MIMCT-Q45) was prepared, and its reliability and validity were confirmed. MIMCT-Q45 might serve as a guidance on basic knowledge to be acquired by students and resident pharmacists and be useful to confirm the level of acquired knowledge.

\section{Introduction}

Reduction of incidents and medical errors involving drugs is an important medical issue ${ }^{1}$ because it has been clarified that drugs are involved in about half of incidents and medical errors at medical practice sites, ${ }^{2,3}$ and prevention of these and minimizing negative influences of these on patients are likely to contribute to medical safety. Especially, many medicine used for cancer treatment, such as anticancer drugs, psychotropic drugs, and narcotics for medical use, have a high risk, ${ }^{4-6}$ for which concentrated measures should be taken.

Measures to improve the safety and quality of cancer treatment, such as regimen registration system, intervention of wards and outpatient chemotherapy rooms by pharmacists, and certification system of pharmacists specialized in the oncology field, have recently penetrated clinical practice in Japan, with which the previous assignment to pharmacists, which are dispensing and drugs based on prescriptions and delivery within the occupational range, has been shifting to a new form being in charge of following patients from before and after drugs administration to outpatient treatment and treatment at home.

However, previous education concerning medical instruments and materials used to administer drugs was weak, and education concerning dealing with problems with equipment during drug administration, selection of medical materials used for drug administration, and medical devices frequently used in home health care (especially palliative care at home) and instruction of patients inevitably depended on physicians and nurses. Since physicians and nurses are not experts of medical instruments, further involvement of pharmacists in handling medical instruments and materials to administer drugs are needed, and educational targets to promote close involvement of pharmacists in medical instruments and materials were set in the revised core curriculum of pharmaceutical education enforced in 2015. However, the specific medical instruments and materials requiring acquisition of basic knowledge are not stated and no scale to measure whether the knowledge is presented. 
Thus, in this study, focusing on medical instruments and materials used for high-risk treatment for cancer, we developed a knowledge test concerning medical instruments and materials requiring acquisition of basic knowledge for students.

\section{Patients And Methods}

1. Preparation of questionnaire and knowledge test

Focus group discussion was performed by participants consisting of one surgical oncologist (MD), 3 nurses with cancerrelated certification, 2 pharmacists, one clinical engineer, and one well experienced and trained researcher (phD) assumed to have abundant experience of cancer treatment, in which specific medical instruments and materials requiring acquisition of basic knowledge and related knowledge were discussed. The discussion facilitated in accordance with the interview guide. The content of the discussion was entirely recorded. The tape was transcribed and converted to text which was returned to and confirmed by participants and subjected to content analysis.

A list of the medical instruments and materials extracted on content analysis was prepared and defined as 'medical instruments and materials requiring acquisition of basic knowledge'. In addition, parts in which basic knowledge to be acquired were spoken by the medical instruments and materials were extracted by meaning and classified by the medical instruments and materials to prepare categories. In addition to 'correct' and 'false', 'I do not know' was set as a choice to exclude unanswered and accidental correct answers as much as possible, and responses to a knowledge test were collected. Students in the 6th year of Faculty of Pharmacy and Pharmaceutical Sciences, University of Toyama, took the prepared pilot knowledge test as a pretest, and a complete version was prepared after discussion among several researchers. In addition, a questionnaire was prepared by adding questions concerning background of respondents.

\section{Evaluation of validity and reliability of the knowledge test}

To investigate reliability of the knowledge test, the questionnaire survey was performed in students twice with a 2-week interval to confirm reliability using the retest method. For the indices of reliability, the interclass correlation coefficient and Cronbach's a were calculated. In addition, using a 2-parameter logistic model of item response theory (IRT) analysis, difficulty and discrimination of each item were estimated. ${ }^{7}$

The surveys were performed in 4-5th year pharmacy school students of nationwide national, public, and private universities in Japan between January 2015 and March 2015. To verify discriminative validity of the knowledge test, ${ }^{8}$ the questionnaire survey was similarly performed in nurses and pharmacists (medical staff) belonging to cancer treatment base hospitals in Toyama, Japan to investigate whether the total score of medical staff is higher than that of students. The questionnaire survey was performed using the anonymous mailing method.

\section{Statistics and analysis}

\section{1) Statistical analysis}

For between-group comparison, the student $t$-test was used setting the significance level at $<0.05$. For the correlation coefficient, Pearson's product-moment correlation coefficient was used. IRT analysis was performed using EasyEstimation version 2.00, ${ }^{9}$ and the other analyses were performed using IBM SPSS version 22 (IBM Japan Ltd., Tokyo).

\section{2) Content analysis}

Content analysis was performed based on the method reported by Krippendorff et al. ${ }^{10}$ Names of medical instruments and materials and related knowledge stated as those 'requiring acquisition of knowledge' and existing reports and their experience (incident reports, etc.) told by the participants of the discussion were extracted regarding a sematic content as one unit. The extracted sematic contents were inductively classified and abstracted following similarity, and several categories (names of medical instruments and materials/ basic knowledge to be acquired) were prepared. The above process was performed 
independently by several researchers. Discussion was repeated until the results became consistent and the final conclusion was reached.

\section{Ethical considerations}

This study was designed and conducted in accordance with the Declaration of Helsinki. The study plan was approved by the Ethics Committee of University of Toyama (RIN26-11). All subjects (medical staff and students) were adults requiring no legal representative. Informed consent to participate in the study was obtained from all 10 participants who participated in the focus group discussions after oral explanation of the content using documents. For the questionnaire survey, a consent form was sent with the questionnaire by postal mail to the subjects, and sending back responses to the questionnaire was regarded as consent to the study.

\section{Results}

1) Preparation of the knowledge test

The 13 types of medical instruments and materials shown in Table 1 were extracted from the focus group discussion by content analysis and listed in the knowledge test.

Table 1

Names of extracted medical instruments and materials

\begin{tabular}{|l|l|}
\hline Medical instruments and materials & Number of statements \\
\hline Pump (common subjects) & 20 \\
\hline Infusion pump & 20 \\
\hline PCA $^{*}$ pump & 11 \\
\hline Infusion line & 9 \\
\hline Syringe pump & 7 \\
\hline Infuser pump & 7 \\
\hline Filter & 5 \\
\hline Injection needle & 3 \\
\hline Central venous port & 2 \\
\hline Syringe & 2 \\
\hline Drip Eye ${ }^{\circledR}$ & 2 \\
\hline Catheter & 1 \\
\hline Cufftee Pump & 1 \\
\hline *PCA: Patient Controlled Analgesia & \\
\hline
\end{tabular}

2) Questionnaire survey

A total of 2,331 questionnaire forms were distributed to 36 universities per survey. Fourteen universities replied (1st: 1,289 forms, 2nd: 1,201 forms), and their consents have been obtained. One university replied only once. The overall questionnaire collection rate was $53.4 \%$. The background of the respondents is shown in Table 2. 
Table 2

Characteristics of Participants

\begin{tabular}{|lll|}
\hline Item & Student & Medical personnel \\
\hline Age (years, mean \pm standard deviation) & $22.4 \pm 0.5$ & $41.2 \pm 5.3$ \\
\hline Sex (n, male/female) & $1139 / 2568$ & $28 / 417$ \\
\hline Type of university (n, national \& public/private) & $841 / 2865$ & \\
\hline Year of school (n, 4th/5th) & $1883 / 1824$ & \\
\hline Experience of practical training at hospital (n, no/yes) & $2355 / 1351$ & \\
\hline Years of clinical experience & & $12.2 \pm 8.7$ \\
\hline Years of cancer treatment experience & & $4.9 \pm 4.6$ \\
\hline Occupation (n, nurse/pharmacist) & $362 / 83$ \\
\hline
\end{tabular}

3) Reliability and validity of the knowledge test (Table 3) 
Table 3

Item analysis and item response theory of MIMCT-Q45

\begin{tabular}{|c|c|c|c|c|c|c|c|c|c|}
\hline \multirow[t]{2}{*}{ Question } & \multirow[t]{2}{*}{$\mathrm{T} / \mathrm{F}$} & \multicolumn{4}{|c|}{ Correct answer (\%) } & \multicolumn{2}{|l|}{ IRT } & \multirow[t]{2}{*}{ ICC } & \multirow{2}{*}{$\begin{array}{l}\text { Cronbach's } \\
\mathbf{a}\end{array}$} \\
\hline & & Students & $\begin{array}{l}\text { Medical } \\
\text { stuff }\end{array}$ & $\begin{array}{l}P \\
\text { value }\end{array}$ & ES & discrimination & difficulty & & \\
\hline $\begin{array}{l}\text { 1. Common } \\
\text { subjects of } \\
\text { electric } \\
\text { (mechanical) } \\
\text { pumps used to } \\
\text { administer } \\
\text { drugs }\end{array}$ & & 39.0 & 51.7 & $<0.001$ & 0.42 & & & 0.86 & 0.68 \\
\hline $\begin{array}{l}\text { q1 Generally, } \\
\text { electric pumps } \\
\text { have alarm } \\
\text { function against } \\
\text { blockade and } \\
\text { bubbles. }\end{array}$ & $\mathrm{T}$ & 47.9 & 91.0 & & & 1.74 & 0.12 & & \\
\hline $\begin{array}{l}\text { q2 Setting } 2 \text { of } \\
\text { variables: the } \\
\text { flow rate, } \\
\text { administration } \\
\text { time, and } \\
\text { planned dose, } \\
\text { subsequently } \\
\text { set the } \\
\text { remaining one } \\
\text { variable. }\end{array}$ & $\mathrm{T}$ & 42.5 & 49.2 & & & 0.98 & 0.38 & & \\
\hline $\begin{array}{l}\text { q3 Most } \\
\text { episodes in } \\
\text { nursing by the } \\
\text { field are related } \\
\text { to injection and } \\
\text { infusion. }\end{array}$ & $\mathrm{T}$ & 37.5 & 44.3 & & & 1.33 & 0.56 & & \\
\hline $\begin{array}{l}\text { q4 Incidents } \\
\text { caused by } \\
\text { mistakes in } \\
\text { setting and } \\
\text { operation of } \\
\text { electric pumps } \\
\text { account for less } \\
\text { than half of } \\
\text { episodes. }\end{array}$ & $\mathrm{F}$ & 27.4 & 19.1 & & & 1.48 & 0.89 & & \\
\hline $\begin{array}{l}\text { q5 Normally, } \\
\text { electric pumps } \\
\text { are equipped } \\
\text { with a free-flow } \\
\text { prevention } \\
\text { device. }\end{array}$ & $\mathrm{T}$ & 39.8 & 55.1 & & & 1.88 & 0.40 & & \\
\hline $\begin{array}{l}\text { 2. Infusion } \\
\text { pump }\end{array}$ & & 41.3 & 61.8 & $<0.001$ & 0.69 & & & 0.89 & 0.69 \\
\hline $\begin{array}{l}\text { q6 There are } \\
\text { dripping- and } \\
\text { volume-control } \\
\text { types of flow } \\
\text { rate control } \\
\text { system of } \\
\text { infusion pump }\end{array}$ & $\mathrm{T}$ & 35.2 & 37.1 & & & 2.15 & 0.56 & & \\
\hline
\end{tabular}

IRT: Item response theory, ICC: Interclass correlation coefficient 


\begin{tabular}{|c|c|c|c|c|c|c|c|c|c|}
\hline \multirow[t]{2}{*}{ Question } & \multirow[t]{2}{*}{$\mathrm{T} / \mathrm{F}$} & \multicolumn{4}{|c|}{ Correct answer (\%) } & \multicolumn{2}{|l|}{ IRT } & \multirow[t]{2}{*}{ ICC } & \multirow{2}{*}{$\begin{array}{l}\text { Cronbach's } \\
\mathbf{a}\end{array}$} \\
\hline & & Students & $\begin{array}{l}\text { Medical } \\
\text { stuff }\end{array}$ & $\begin{array}{l}P \\
\text { value }\end{array}$ & ES & discrimination & difficulty & & \\
\hline $\begin{array}{l}\text { q7 There are } \\
\text { stationary and } \\
\text { portable types } \\
\text { corresponding } \\
\text { to the use } \\
\text { condition of } \\
\text { infusion pump. }\end{array}$ & $\mathrm{T}$ & 49.0 & 58.4 & & & 1.95 & 0.05 & & \\
\hline $\begin{array}{l}\text { q8 Infusion } \\
\text { pumps send } \\
\text { solution by } \\
\text { pressing the } \\
\text { tube set in the } \\
\text { unit. }\end{array}$ & $\mathrm{T}$ & 15.9 & 58.7 & & & 1.02 & 1.88 & & \\
\hline $\begin{array}{l}\text { q9 Uncleanness } \\
\text { in the pump unit } \\
\text { has a negative } \\
\text { influence on the } \\
\text { accuracy of flow } \\
\text { rate. }\end{array}$ & $\mathrm{T}$ & 72.4 & 87.6 & & & 1.80 & -0.91 & & \\
\hline $\begin{array}{l}\text { q10 The cover } \\
\text { of infusion } \\
\text { pumps equipped } \\
\text { with a free-flow } \\
\text { prevention } \\
\text { device can be } \\
\text { opened while } \\
\text { the clamp is } \\
\text { open. }\end{array}$ & $\mathrm{F}$ & 34.1 & 67.2 & & & 2.03 & 0.51 & & \\
\hline 3. Syringe pump & & 38.0 & 64.4 & $<0.001$ & 0.87 & & & 0.86 & 0.64 \\
\hline $\begin{array}{l}\text { q11 Generally, } \\
\text { the injection } \\
\text { accuracy of } \\
\text { syringe pumps } \\
\text { is higher than } \\
\text { that of infusion } \\
\text { pumps. }\end{array}$ & $\mathrm{T}$ & 39.5 & 81.8 & & & 1.19 & 0.46 & & \\
\hline $\begin{array}{l}\text { q12 Syringe } \\
\text { pumps are } \\
\text { suitable for } \\
\text { injection of a } \\
\text { large volume of } \\
\text { infusion } \\
\text { solution. }\end{array}$ & $\mathrm{F}$ & 57.7 & 93.0 & & & 1.39 & -0.34 & & \\
\hline $\begin{array}{l}\text { q13 Drug } \\
\text { solution is not } \\
\text { injected unless } \\
\text { the plunger is } \\
\text { completely set. }\end{array}$ & $\mathrm{F}$ & 18.7 & 29.2 & & & 1.06 & 1.54 & & \\
\hline
\end{tabular}

IRT: Item response theory, ICC: Interclass correlation coefficient 


\begin{tabular}{|c|c|c|c|c|c|c|c|c|c|}
\hline \multirow[t]{2}{*}{ Question } & \multirow[t]{2}{*}{$\mathrm{T} / \mathrm{F}$} & \multicolumn{4}{|c|}{ Correct answer (\%) } & \multicolumn{2}{|l|}{ IRT } & \multirow[t]{2}{*}{ ICC } & \multirow{2}{*}{$\begin{array}{l}\text { Cronbach's } \\
\mathbf{a}\end{array}$} \\
\hline & & Students & $\begin{array}{l}\text { Medical } \\
\text { stuff }\end{array}$ & $\begin{array}{l}P \\
\text { value }\end{array}$ & ES & discrimination & difficulty & & \\
\hline $\begin{array}{l}\text { q14 Solution is } \\
\text { continuously } \\
\text { injected by } \\
\text { setting a rigid } \\
\text { syringe and } \\
\text { pressing the } \\
\text { plunger at a } \\
\text { constant speed. }\end{array}$ & $\mathrm{T}$ & 36.1 & 53.5 & & & 1.98 & 0.54 & & \\
\hline 4. Infuser pump & & 33.1 & 48.3 & $<0.001$ & 0.45 & & & 0.88 & 0.75 \\
\hline $\begin{array}{l}\text { q15 Infuser } \\
\text { pumps } \\
\text { continuously } \\
\text { administer drug } \\
\text { solution using a } \\
\text { balloon, } \\
\text { atmosphere, or } \\
\text { spring as a } \\
\text { driving force. }\end{array}$ & $\mathrm{T}$ & 41.8 & 59.3 & & & 2.55 & 0.34 & & \\
\hline $\begin{array}{l}\text { q16 Infuser } \\
\text { pumps are used } \\
\text { for continuous } \\
\text { administration } \\
\text { of anticancer } \\
\text { drugs and } \\
\text { narcotics for } \\
\text { medial use. }\end{array}$ & $\mathrm{T}$ & 43.3 & 67.6 & & & 2.14 & 0.29 & & \\
\hline $\begin{array}{l}\text { q17 Since } \\
\text { infuser pumps } \\
\text { are equipped } \\
\text { with a flow rate- } \\
\text { control system, } \\
\text { the viscosity } \\
\text { and temperature } \\
\text { of drug } \\
\text { solutions do not } \\
\text { influence the } \\
\text { flow rate. }\end{array}$ & $\mathrm{F}$ & 41.0 & 52.1 & & & 2.30 & 0.35 & & \\
\hline $\begin{array}{l}\text { q18 Flow rate- } \\
\text { control systems } \\
\text { have a function } \\
\text { to remove a } \\
\text { trace amount of } \\
\text { bubbles to } \\
\text { prevent them } \\
\text { from entering } \\
\text { the patient's } \\
\text { body. }\end{array}$ & $\mathrm{F}$ & 6.4 & 14.2 & & & 1.15 & 2.69 & & \\
\hline 5. PCA pump & & 24.0 & 34.3 & $<0.001$ & 0.39 & & & 0.89 & 0.58 \\
\hline
\end{tabular}

IRT: Item response theory, ICC: Interclass correlation coefficient 


\begin{tabular}{|c|c|c|c|c|c|c|c|c|c|}
\hline \multirow[t]{2}{*}{ Question } & \multirow[t]{2}{*}{$\mathrm{T} / \mathrm{F}$} & \multicolumn{4}{|c|}{ Correct answer (\%) } & \multicolumn{2}{|l|}{ IRT } & \multirow[t]{2}{*}{ ICC } & \multirow{2}{*}{$\begin{array}{l}\text { Cronbach's } \\
\text { a }\end{array}$} \\
\hline & & Students & $\begin{array}{l}\text { Medical } \\
\text { stuff }\end{array}$ & $\begin{array}{l}P \\
\text { value }\end{array}$ & ES & discrimination & difficulty & & \\
\hline $\begin{array}{l}\text { q19 PCA is an } \\
\text { abbreviation of } \\
\text { Pain Care } \\
\text { Adjustment } \\
\text { Method } \\
\text { representing a } \\
\text { method for } \\
\text { physicians to } \\
\text { flexibly adjust } \\
\text { the dose of } \\
\text { analgesics. }\end{array}$ & $\mathrm{F}$ & 7.1 & 6.5 & & & 1.02 & 3.00 & & \\
\hline $\begin{array}{l}\text { q20 Using a PCA } \\
\text { pump, patients } \\
\text { can administer } \\
\text { injection } \\
\text { (analgesics) in } \\
\text { their judgment } \\
\text { when they feel } \\
\text { pain. }\end{array}$ & $\mathrm{T}$ & 37.3 & 72.4 & & & 1.45 & 0.65 & & \\
\hline $\begin{array}{l}\text { q21 In electric } \\
\text { PCA pumps, } \\
\text { complex } \\
\text { administration } \\
\text { methods can be } \\
\text { programmed } \\
\text { and history of } \\
\text { administration } \\
\text { can be recorded. }\end{array}$ & $\mathrm{T}$ & 33.4 & 31.5 & & & 2.11 & 0.61 & & \\
\hline $\begin{array}{l}\text { q22 In balloon- } \\
\text { type PCA } \\
\text { pumps, the } \\
\text { lockout time and } \\
\text { flow rate can be } \\
\text { freely changed. }\end{array}$ & $\mathrm{F}$ & 18.2 & 27.0 & & & 1.80 & 1.33 & & \\
\hline $\begin{array}{l}\text { 6. Drug injection } \\
\text { controller (Drip } \\
\text { Eyeß) }\end{array}$ & & 16.1 & 12.1 & 0.004 & 0.16 & & & 0.92 & 0.68 \\
\hline $\begin{array}{l}\text { q23 Drug } \\
\text { injection } \\
\text { controller is a } \\
\text { dripping control- } \\
\text { type infusion } \\
\text { pump. }\end{array}$ & $\mathrm{F}$ & 5.6 & 3.6 & & & 1.57 & 2.30 & & \\
\hline $\begin{array}{l}\text { q24 Similar to } \\
\text { volume control- } \\
\text { type infusion } \\
\text { pumps, it has an } \\
\text { alarm function } \\
\text { against } \\
\text { blockade and } \\
\text { free flow- } \\
\text { preventive } \\
\text { function. }\end{array}$ & $\mathrm{T}$ & 24.8 & 16.0 & & & 3.09 & 0.80 & & \\
\hline
\end{tabular}

IRT: Item response theory, ICC: Interclass correlation coefficient 


\begin{tabular}{|c|c|c|c|c|c|c|c|c|c|}
\hline \multirow[t]{2}{*}{ Question } & \multirow[t]{2}{*}{$\mathrm{T} / \mathrm{F}$} & \multicolumn{4}{|c|}{ Correct answer (\%) } & \multicolumn{2}{|l|}{ IRT } & \multirow[t]{2}{*}{ ICC } & \multirow{2}{*}{$\begin{array}{l}\text { Cronbach's } \\
\mathbf{a}\end{array}$} \\
\hline & & Students & $\begin{array}{l}\text { Medical } \\
\text { stuff }\end{array}$ & $\begin{array}{l}P \\
\text { value }\end{array}$ & ES & discrimination & difficulty & & \\
\hline $\begin{array}{l}\text { q25 Compared } \\
\text { with volume } \\
\text { control-type } \\
\text { infusion pumps, } \\
\text { it reduces the } \\
\text { risk of } \\
\text { extravasation. }\end{array}$ & $\mathrm{T}$ & 22.6 & 15.7 & & & 2.75 & 0.89 & & \\
\hline $\begin{array}{l}\text { q26 Drug } \\
\text { injection } \\
\text { controller can } \\
\text { also be used for } \\
\text { precision } \\
\text { microinjection, } \\
\text { arterial } \\
\text { administration, } \\
\text { and blood } \\
\text { transfusion. }\end{array}$ & $\mathrm{F}$ & 11.6 & 13.0 & & & 2.18 & 1.50 & & \\
\hline $\begin{array}{l}\text { 7. Infusion line } \\
\text { (infusion } \\
\text { set/drip infusion } \\
\text { line) }\end{array}$ & & 36.4 & 54.2 & $<0.001$ & 0.69 & & & 0.90 & 0.81 \\
\hline $\begin{array}{l}\text { q27 Infusion } \\
\text { lines represent } \\
\text { medical } \\
\text { instruments to } \\
\text { inject infusion } \\
\text { solution into } \\
\text { patients. }\end{array}$ & $\mathrm{T}$ & 63.4 & 90.3 & & & 1.54 & -0.54 & & \\
\hline $\begin{array}{l}\text { q28 Infusion } \\
\text { lines are } \\
\text { comprised of } \\
\text { bottle needles, } \\
\text { drip tubes, } \\
\text { clamps, and } \\
\text { connecting } \\
\text { parts. }\end{array}$ & $\mathrm{T}$ & 53.2 & 79.3 & & & 2.05 & -0.07 & & \\
\hline $\begin{array}{l}\text { q29 A bottle } \\
\text { needle is } \\
\text { connected to the } \\
\text { end of the } \\
\text { infusion line and } \\
\text { inserted into } \\
\text { patients to fix } \\
\text { the line. }\end{array}$ & $\mathrm{F}$ & 15.1 & 57.1 & & & 1.19 & 1.76 & & \\
\hline $\begin{array}{l}\text { q30 There are } \\
\text { various types of } \\
\text { infusion line, } \\
\text { such as those } \\
\text { for general use, } \\
\text { adults, and } \\
\text { blood } \\
\text { transfusion. }\end{array}$ & $\mathrm{T}$ & 34.3 & 59.8 & & & 1.29 & 0.59 & & \\
\hline
\end{tabular}

IRT: Item response theory, ICC: Interclass correlation coefficient 


\begin{tabular}{|c|c|c|c|c|c|c|c|c|c|}
\hline \multirow[t]{2}{*}{ Question } & \multirow[t]{2}{*}{$\mathrm{T} / \mathrm{F}$} & \multicolumn{4}{|c|}{ Correct answer (\%) } & \multicolumn{2}{|l|}{ IRT } & \multirow[t]{2}{*}{ ICC } & \multirow{2}{*}{$\begin{array}{l}\text { Cronbach's } \\
\mathbf{a}\end{array}$} \\
\hline & & Students & $\begin{array}{l}\text { Medical } \\
\text { stuff }\end{array}$ & $\begin{array}{l}P \\
\text { value }\end{array}$ & ES & discrimination & difficulty & & \\
\hline $\begin{array}{l}\text { q31 The same } \\
\text { infusion line can } \\
\text { be used for } \\
\text { infusion by } \\
\text { natural dropping } \\
\text { and using an } \\
\text { infusion pump. }\end{array}$ & $\mathrm{F}$ & 36.0 & 61.6 & & & 1.78 & 0.52 & & \\
\hline $\begin{array}{l}\text { q32 A metal } \\
\text { needle is used in } \\
\text { a drip tube of a } \\
1-\mathrm{mL}=20 \text { drops } \\
\text { type infusion } \\
\text { line, for which } \\
\text { attention should } \\
\text { be paid to } \\
\text { hydrophilization. }\end{array}$ & $\mathrm{F}$ & 7.1 & 40.9 & & & 1.48 & 2.25 & & \\
\hline $\begin{array}{l}\text { q33 Plasticizer } \\
\text { is added to } \\
\text { soften the tube } \\
\text { material for } \\
\text { easy molding. }\end{array}$ & $\mathrm{T}$ & 35.5 & 29.0 & & & 1.53 & 0.61 & & \\
\hline $\begin{array}{l}\text { q34 Regarding } \\
\text { inscription of } \\
\text { tube materials, } \\
\text { the meanings of } \\
\text { PVC-Free and } \\
\text { DEHP-Free are } \\
\text { the same. }\end{array}$ & $\mathrm{F}$ & 31.8 & 33.5 & & & 1.89 & 0.68 & & \\
\hline $\begin{array}{l}\text { q35 Joints of an } \\
\text { infusion line } \\
\text { forming a dead } \\
\text { space cause } \\
\text { infection. }\end{array}$ & $\mathrm{T}$ & 55.7 & 68.1 & & & 2.46 & -0.13 & & \\
\hline $\begin{array}{l}\text { q36 Products in } \\
\text { which } \\
\text { polycarbonate is } \\
\text { used for the } \\
\text { joints may get } \\
\text { cracked } \\
\text { depending on } \\
\text { the drug } \\
\text { infusion } \\
\text { administered. }\end{array}$ & $\mathrm{T}$ & 32.0 & 22.2 & & & 2.55 & 0.62 & & \\
\hline 8. Filter & & 27.6 & 22.1 & $<0.001$ & 0.21 & & & 0.86 & 0.63 \\
\hline $\begin{array}{l}\text { q37 A filter } \\
\text { incorporated in } \\
\text { an infusion line } \\
\text { is termed in-line } \\
\text { filter. }\end{array}$ & $\mathrm{T}$ & 48.6 & 61.3 & & & 2.11 & 0.09 & & \\
\hline $\begin{array}{l}\text { q38 To remove } \\
\text { bacteria, a 0.45- } \\
\mu \text { filter with a } \\
\text { homogeneous } \\
\text { pore size is } \\
\text { used. }\end{array}$ & $\mathrm{F}$ & 21.4 & 10.6 & & & 1.18 & 1.49 & & \\
\hline
\end{tabular}




\begin{tabular}{|c|c|c|c|c|c|c|c|c|c|}
\hline \multirow[t]{2}{*}{ Question } & \multirow[t]{2}{*}{$\mathrm{T} / \mathrm{F}$} & \multicolumn{4}{|c|}{ Correct answer (\%) } & \multicolumn{2}{|l|}{ IRT } & \multirow[t]{2}{*}{ ICC } & \multirow{2}{*}{$\begin{array}{l}\text { Cronbach's } \\
\text { a }\end{array}$} \\
\hline & & Students & $\begin{array}{l}\text { Medical } \\
\text { stuff }\end{array}$ & $\begin{array}{l}P \\
\text { value }\end{array}$ & ES & discrimination & difficulty & & \\
\hline $\begin{array}{l}\text { q39 To remove } \\
\text { glass fragments } \\
\text { produced by } \\
\text { cutting an } \\
\text { ampule, a 0.8- } \\
\mu \mathrm{m} \text { filter is used. }\end{array}$ & $\mathrm{T}$ & 25.2 & 14.8 & & & 1.64 & 1.01 & & \\
\hline $\begin{array}{l}\text { q40 When } \\
\text { clogging of } \\
\text { filters by } \\
\text { changes in } \\
\text { compounding of } \\
\text { infusion } \\
\text { solution is } \\
\text { concerned, no } \\
\text { filter is used. }\end{array}$ & $\mathrm{F}$ & 23.2 & 17.8 & & & 1.20 & 1.36 & & \\
\hline $\begin{array}{l}\text { q41 Filters with } \\
\text { a function to } \\
\text { remove air } \\
\text { mixed in } \\
\text { infusion } \\
\text { solution } \\
\text { comprised of } \\
\text { only a } \\
\text { hydrophobic } \\
\text { membrane. }\end{array}$ & $\mathrm{F}$ & 19.6 & 5.8 & & & 2.22 & 1.10 & & \\
\hline $\begin{array}{l}\text { 9. Other medical } \\
\text { supplies } \\
\text { (injection needle, } \\
\text { injection syringe, } \\
\text { CV port, catheter } \\
\text { etc.) }\end{array}$ & & 23.2 & 29.6 & $<0.001$ & 0.24 & & & 0.90 & 0.56 \\
\hline $\begin{array}{l}\text { q42 When drug } \\
\text { solution is } \\
\text { removed from } \\
\text { an anticancer } \\
\text { drug vial, a } \\
\text { regular bevel } \\
\text { needle is used. }\end{array}$ & $\mathrm{F}$ & 12.6 & 5.8 & & & 1.31 & 1.87 & & \\
\hline $\begin{array}{l}\text { q43 For } \\
\text { puncture into } \\
\text { the port of the } \\
\text { central vein, a } \\
\text { Huber-pointed } \\
\text { needle is used. }\end{array}$ & $\mathrm{T}$ & 19.2 & 40.7 & & & 2.81 & 1.04 & & \\
\hline $\begin{array}{l}\text { q44 When an } \\
\text { anticancer drug } \\
\text { is handled, a slip } \\
\text { tip-type syringe } \\
\text { is used. }\end{array}$ & $\mathrm{F}$ & 13.0 & 13.7 & & & 1.35 & 1.77 & & \\
\hline
\end{tabular}

IRT: Item response theory, ICC: Interclass correlation coefficient 


\begin{tabular}{|c|c|c|c|c|c|c|c|c|c|}
\hline \multirow[t]{2}{*}{ Question } & \multirow[t]{2}{*}{$\mathrm{T} / \mathrm{F}$} & \multicolumn{4}{|c|}{ Correct answer (\%) } & \multicolumn{2}{|l|}{ IRT } & \multirow[t]{2}{*}{ ICC } & \multirow{2}{*}{$\begin{array}{l}\text { Cronbach's } \\
\mathbf{a}\end{array}$} \\
\hline & & Students & $\begin{array}{l}\text { Medical } \\
\text { stuff }\end{array}$ & $\begin{array}{l}\mathrm{P} \\
\text { value }\end{array}$ & ES & discrimination & difficulty & & \\
\hline $\begin{array}{l}\text { q45 To prevent } \\
\text { exposure to } \\
\text { anticancer } \\
\text { drugs, it is } \\
\text { desirable to use } \\
\text { closed } \\
\text { adjustment } \\
\text { instruments and } \\
\text { exclusive } \\
\text { infusion line. }\end{array}$ & $\mathrm{T}$ & 48.1 & 58.0 & & & 2.02 & 0.17 & & \\
\hline Overall & & 32.0 & 43.9 & $<0.001$ & 0.56 & & & 0.90 & 0.94 \\
\hline
\end{tabular}

Overall, both Cronbach's a and the interclass correlation coefficient were high, but Cronbach's a of 'PCA pump' and 'other medical materials' was slightly low (0.56-0.58).

The mean score of the medical personnel (nurses and pharmacists) widely varied and biases of knowledge were present, whereas the score of the students was consistently lower significantly than that of the medical staff, and the overall effect size was 0.56 (medium).

The discrimination estimated by IRT ranged from 0.98 to 3.09 , and that of difficulty ragged from -0.91 to 3.00 . The total score tended to be higher in respondents who could correctly answer to questions with high discrimination.

\section{Discussion}

A knowledge test concerning medical instruments and materials of cancer treatment (medical instruments and materials for cancer treatment- Questionnaire 45 (MIMCT-Q45)) was prepared and its reliability and validity were confirmed. MIMCT-Q45, prepared focusing on medical instruments and materials used for high-risk cancer treatment including palliative care at home, may serve as a guidance on basic knowledge to be acquired by students and resident pharmacists and be useful to confirm the level of acquired knowledge.

In the responses from medical personnel collected as a positive control, the percentage of the score was high in items frequently used in routine practice, such as infusion pumps and syringe pumps, but it was low in some domains of items less frequently used, such as home health care-specific devices and materials, and the rate of correct answer was lower than $10 \%$ in 3 questions in the medical staff. Since MIMCT-Q45 is comprised of items considered important by experts of cancer treatment, it was concluded that a low percentage of the score in the medical staff does not indicate that the knowledge is unnecessary. In particular, it might be useful to incorporate it into the educational curriculum of physicians.

The reason for the bias and variation in knowledge among the medical professionals was presumably due to the differences in the types of work they had experienced. The average age of the medical professionals in this study was about 40 years old, but the medical education that these people had received in Japan did not include a curriculum for learning about medical instrument and materials. Hence, they had to acquire the knowledge through hands-on experience. In particular, it is only recently that pharmacists have become involved in cancer chemotherapy and palliative medicine. In addition, the work involved in these areas is subdivided (e.g., those in charge of compounding anticancer drugs, those in charge of giving medication guidance in the wards, those in charge of giving medication guidance in outpatient clinics, and those in charge of home care), and the division of work is clearly defined, and the work assignment is hardly ever changed. It is suggested that this background of the medical field might have influenced the bias in knowledge. 
In contrast, the percentage of the score in the students was lower than that in the medical staff in most items, suggesting that discriminative validity was verified. The percentage of the score was higher in the students than in the medical staff in a few items. These may have been unfamiliar medical instruments and materials even for medical staff, suggesting the necessity of on the-job-education.

The importance of knowledge related to infusion devices for home health care requiring close involvement of medical staff has also been pointed out, ${ }^{11,12}$ but it was discussed from viewpoints of physicians and nurses in many cases, in which greater importance is attached to training and knowledge concerning administration of transfusion and drugs. ${ }^{13}$ In Japan, pharmacists do not have a right to prescribe or directly administer drugs in response to instruction from physicians, for which required knowledge and training programs (interactions between infusion devices and drugs and troubleshooting) may be different. Accordingly, the systematically constructed knowledge required for resident pharmacists and pharmacy students may be the important first step toward providing safe medical care.

There were several limitations of this study. Since MIMCT-Q45 was prepared aiming at widely measuring knowledge concerning several medical instruments and materials, important education items may have been overlooked. However, the amount of required knowledge concerning only infusion devices is too large to cover entire knowledge. Therefore, this knowledge test on limited targets may be useful to measure the educational effect. Since MIMCT-Q45 was developed mainly targeting Japanese pharmacy school students, it was prepared so as to match the medical state in Japan. Accordingly, to apply it to other countries and occupations, modification may be necessary in several items.

\section{Conclusion}

In conclusion, MIMCT-Q45 targeting pharmacy students and resident pharmacists was developed. Development of educational materials concerning 'medical instruments and materials requiring acquisition of knowledge' and measurement of its educational effect using MIMCT-Q45 remain as tasks.

\section{Abbreviations}

MIMCT-Q45

medical instruments and materials for cancer treatment-Questionnaire 45

IRT

item response theory

\section{Declarations}

\section{Acknowledgements}

This study was performed in 2013-2014 as 'Verification of the learning effect and usefulness concerning video educational materials for learning medical instruments and materials' in 'Project for promotion of medical professionals training at Pharmacy School from the Ministry of Education, Culture, Sports, Science and Technology. We are grateful to faculty members and students for their cooperation.

\section{Author contributions}

$\mathrm{KT}$ and $\mathrm{KH}$ made a substantial contribution to the concept or design of the work; or acquisition, analysis or interpretation of data. KT, AN, HO, and HY drafted the article or revised it critically for important intellectual content. KT, HO, and $\mathrm{KH}$ have participated. All authors approved the version to be published.

\section{Ethical approval}

This study plan was approved by the Ethics Committee of University of Toyama (RIN26-11). 


\section{Conflicts of interests}

The authors declare no competing interests.

\section{References}

1. Kawamura, H. The analysis of Hiyari-Hatto reports for prevention of medication errors and falls. J Jpn Soc Health Care Manage, 2, 265-269 (2002).

2. Funakoshi, S. et al. The Effect of Grasp and Consideration of Medication-related incidents on Medical Safety by Pharmacists in the Ward. J Jpn Soc Hosp Pharm, 50, 853-857 (2014).

3. Ministry of Health, Labour and Welfare (Available at) http://www1.mhlw.go.jp/houdou/1206/h0626-1_10.html. Data accessed: November 10, 2017

4. Sugiura, S., Gohma, H. \& Hamajima, N. Measures of Preventing Occupational Exposure to Hazardous Drugs - Based on New Insights. Jpn J Cancer Chemother, 41, 923-925 (2014).

5. Amino, Y. et al. The Management of Chemotherapy Regimen on the Electronic Medical Record. Jpn J Cancer Chemother, 41, 1135-1138 (2014).

6. Yamamot, H., Kitada, N., Shibatani, N., Hirabatake, M. \& Hashida, T. Role of Pharmacists in Introduction of New Drugs for the Minimization of Risk - In Case of Denosumab. Jpn J Drug Inform, 16, 28-32 (2014).

7. Streiner, D. Health Measurement Scales: A Practical Guide to Their Development and Use. 4th edition: Oxford University Press, New York, 2008

8. Murayama, K. Validity. Historical and Psychometric Perspectives. The Annual Report of Educational Psychology in Japan. 2012; 51: 118-130

9. Kumagai, R. GUI Development of IRT analysis programs for beginners: EasyEstimation series. Jpn J Res Testing, 5, 107118 (2009).

10. Krippendorff, K. Content Analysis: an Introduction to Its Methodology. 1st edition: Sage Publications, Beverly Hills, 1980

11. Beer, J. M., McBride, S. E., Mitzner, T. L. \& Rogers, W. A. Understanding challenges in the front lines of home health care: a human-systems approach. Appl Ergon, 45, 1687-1699 (2014).

12. Lyons, I. \& Blandford, A. Safer healthcare at home: Detecting, correcting and learning from incidents involving infusion devices. Appl Ergon, 67, 104-114 (2018).

13. Kim, U. R., Peterfreund, R. A. \& Lovich, M. A. Drug Infusion Systems: Technologies, Performance, and Pitfalls. Anesth Analg, 124, 1493-1505 (2017).

\section{Supplementary Files}

This is a list of supplementary files associated with this preprint. Click to download.

- KouichiTanabeEngverAMedicalinstrumentsandmaterialsforcancertreatmentQuestionnaire45MIMCTQ45.pdf

- KouichiTanabeJpnverAMedicalinstrumentsandmaterialsforcancertreatmentQuestionnaire45MIMCTQ45.pdf 